\title{
CIENTIIFICAS E INVENTORAS A TRAVÉS DE LOS CUENTOS
}

\section{FEMALE SCIENTISTS AND INVENTORS THROUGH TALES}

María Encina Calvo Iglesias. Universidad de Santiago de Compostela-España

encina.calvo@usc.es

Resumen: En los últimos años, en nuestro país se han llevado a cabo distintas iniciativas para atraer alumnas al ámbito STEM (Science, Technology, Engineering and Mathematics), donde continúan siendo una minoría. En esta comunicación, mostramos una selección de científicas e inventoras cuyas biografías aparecen en cuentos y relatos dirigidos al público infantil y juvenil y que ha formado parte del material complementario del programa Unha enxeñeira ou científica en cada cole, cuyo objetivo es visibilizar a científicas y tecnólogas en colegios de primaria. Estos cuentos y relatos podrían utilizarse para mostrar las contribuciones de las mujeres al avance de la ciencia y tecnología, combatiendo la cultura androcéntrica que aún predomina en los libros de texto escolares y proporcionando referentes femeninos en el ámbito científico-tecnológico, algo de gran importancia en la elección de estudios.

Palabras clave cuentos, estereotipos de género, igualdad, ciencia y tecnología, educación primaria. 
Abstract In recent years, different initiatives have been carried out in our country to attract girls to the STEM disciplines (Science, Technology, Engineering and Mathematics), where they continue to be a minority. In this communication, we show a selection of female scientists and inventors whose biographies appear in tales and stories aimed at children and young people, and have been part of the complementary material of the program Unha enxeñeira ou científica en cada cole, whose objective is to make female scientists and technologists visible in primary schools. These stories and tales could be used to show the contributions of women to the advancement of science and technology, fighting the androcentric culture that still predominates in school textbooks and providing feminine references in the scientifictechnological field, something of great importance in the choice of studies.

Keywords tales, gender stereotypes, equality, science and technology, primary education.

\section{Introducción}

En nuestro país, las mujeres son mayoría entre el alumnado universitario, pero continúan siendo una minoría dentro de las carreras científico-técnicas, también llamadas STEM por sus acrónimos en inglés (Science, Technology, Engineering and Mathematics). Esta baja representación femenina no se debe a un menor rendimiento o habilidades de las chicas en estos ámbitos, sino a la elección durante su trayectoria escolar que está muy marcada por los estereotipos de género (Hill, Corbett y Rose, 2010; Couso, 2015). Además, estos estereotipos afectan desde edades tempranas (Bian, Leslie y Cimpian, 2017), como nos muestra el video realizado recientemente por la ONG Liga de la Educación donde se pregunta a niños y niñas que quieren ser de mayores (Agudo, 2018), y desaniman a las mujeres a 
seguir determinadas carreras universitarias (Miller, Eagly y Linn, 2015). Esta baja representación femenina en el ámbito STEM perjudica a las mujeres, ya que reduce sus oportunidades de trabajo y de participación en los avances y las decisiones del futuro tecnológico, y a toda la sociedad porque si el ámbito STEM no es capaz de atraer a todo el talento, no podrá disponer de toda la diversidad que podría fomentar la innovación. Así, los estereotipos de género siguen condicionando la elección de estudios y "frente a ello, la escuela no logra atraer y retener a las niñas y chicas en estos estudios, es decir, está fallando en su labor de fomentar la igualdad" (Fernández e lbáñez, 2018).

Una de las causas de esta brecha de género es la falta de referentes femeninos que sirvan de ejemplo a las futuras ingenieras, tecnólogas o científicas, tanto en el ámbito profesional como en el académico. A esta falta de modelos contribuyen los libros de texto, ya que en los textos escolares habituales no se recoge de forma adecuada la importante contribución de las mujeres a la ciencia y tecnología (Saéz y Clavero, 2016). También es necesaria más formación en género del profesorado ya que "es fundamental para frenar las desigualdades, la discriminación y la violencia hacia la mujer..." (Pinedo, Arroyo y Berzosa, 2018). Y una mayor visibilización en los medios de comunicación, ya que las mujeres científicas españolas son invisibles en la prensa (Aladro y col., 2014; García-Nieto, 2013), y su imagen se usa mucho más como adorno que los hombres en textos que tratan de ciencia (González y col., 2017; Angulo, 2018).

En los últimos años, se han llevado a cabo distintas 
iniciativas para atraer alumnas a las disciplinas STEM en nuestro país, entre ellas el programa Unha enxeñeira ou científica en cada cole (en español, Una ingeniera o científica en cada cole). Una actividad que pretende visibilizar a las ingenieras y científicas para crear referentes femeninos e incentivar la presencia de chicas en carreras relacionadas con las disciplinas STEM (Aguayo y Calvo, 2016; Calvo y col., 2017). En la segunda edición de esta actividad, como material complementario a los talleres impartidos por científicas e ingenieras, se elaboró un documento con referencias a cuentos y relatos sobre mujeres que han contribuido al avance de la ciencia y tecnología y que describimos con más detalle en la siguiente sección.

\section{Científicas e inventoras en los libros texto}

El libro de texto es "la herramienta a partir de la cual se reproduce, en la escuela el conocimiento científico, o sea el caudal de saberes culturales que el curriculum escolar selecciona y ordena para su transmisión y aprendizaje" (Lomas, citado por López-Navajas 2014). Por ello desde finales de los años setenta del siglo $X X$, los sesgos sexistas de los libros de texto han sido el objetivo de numerosas investigaciones (Álvarez y Soneira, 1991; Subirats, 1993; Blanco, 2000; Manassero y Vázquez, 2003; Hamodi, 2014; López-Navajas, 2014; Álvarez y col., 2017). Sin embargo, como señala Vaillo (2016) "los resultados de las investigaciones emprendidas continúan dibujando una situación muy poco halagüeña en cuanto a la incorporación de la igualdad" y "los hombres y mujeres del futuro reciben 
todavía un conocimiento amputado, un relato histórico incompleto basado en un canon cultural que se corresponde a los valores y espacios masculinos, y no a los de la totalidad de la población" (Rodríguez-Pina, 2018).

La ausencia de mujeres científicas en los libros de ciencias y tecnología, ya señalada por Álvarez y Soneira (1991) o Manassero y Vázquez (2003), parece mantenerse a pesar de que la legislación vigente reconoce la igualdad de género como uno de los objetivos de la Enseñanza en Educación Primaria (Ley Orgánica para la Mejora de la Calidad Educativa 8/2013, artículos 3 y 10). Recientemente, Navarro (2016) analizó los libros de educación primaria del área de Ciencias Naturales de dos editoriales de ámbito nacional, Anaya y Santillana. Los resultados mostraron que la presencia de mujeres científicas es muy escasa, en concreto en los libros de Santillana sólo aparece Marie Curie frente a 17 científicos o inventores y en el caso de la editorial Anaya además de Marie Curie también se cita a la inventora Mary Anderson frente a 8 científicos e inventores.

En ambas editoriales, es en el libro de cuarto de primaria donde se mencionan más personalidades célebres, sin embargo, en otras editoriales como SM es en el tercer ciclo de primaria donde se concentran las biografías de científicos e inventores, en la sección Locos por la ciencia donde no se nombran las contribuciones científicas o inventos de mujeres. Esta baja presencia femenina en los libros de texto continúa en la ESO, donde Gutiérrez (2017) ha constatado que las mujeres no aparecen en los libros de Tecnología.

Por ello, con el fin de saber qué conocimientos sobre científicas e inventoras podía tener el alumnado al que iban 
dirigidos los talleres de la actividad Unha enxeñeira ou científica en cada cole, se analizaron los libros de texto de sexto de primaria de uno de los colegios participantes, en particular los de sexto curso. Así, nos encontramos que en el libro de Ciencias da Natureza de la editorial SM aparece una sección Tolos pola ciencia (en castellano, Locos por la ciencia), donde en formato cómic se relata la vida de Pasteur y Van Leeuwenhoek, Servet, Semmelweis, Fleming, Edison y Lavoisier, aunque en las viñetas aparece trabajando con su mujer Marie Paulze, conocida como la "madre de la química moderna" (Macho, 2016) y a quien deberían haber incluido como científica. En el libro de Lingua (Lengua Gallega) de la editorial Anaya aparece una sección Elas contan (Ellas cuentan) con una breve biografía de mujeres deportistas, pintoras, y científicas como la oceonógrafa Anxeles Alvariño, la primera y única mujer a quien dedicaron el Día de la Ciencia en Galicia, la ornitóloga Ruth Muñiz directora de un centro de recuperación del Águila Harpía y la bióloga Beatriz Pereira, responsable técnica del acuario en Dubai. En el de inglés TigerTracks de MacMillan dedican el tema 5 a los inventos, y mencionan a cuatro inventores entre los cuales aparece Josephine Cochrane, la inventora del lavaplatos, en la página 45. También aparece Marie Curie en la página 49 con dos inventores. Sin embargo, la lectura principal en formato cómic se la dedican a Edward Jenner, sin mencionar a lady Montagu como precursora de la vacuna de la viruela en Inglaterra o a Isabel Zendal, quien tuvo un importante papel en la Expedición Filantrópica contra la viruela y sobre la que ya se han escrito dos novelas: A flor de piel y Los niños de la viruela. 
Este análisis nos lleva a las mismas conclusiones que las señaladas por la directora de la fundación búlgara Investigación y Tecnología para la Educación de Género con respecto a los libros de texto, ya que "parece que todos los avances e inventos los han hecho hombres. De tal modo, que los alumnos se creen que ellos, los varones, han creado todo y son capaces de todo. Y las niñas acaban creyendo que ellas no valen" (Agudo, 2018).

Por ello, no deberíamos extrañarnos que cuando se pide a un grupo de escolares que se imagine a una persona que trabaja en ciencia y lo dibuje en su ambiente de trabajo, los resultados suelan ser similares: un varón, a menudo calvo o con el pelo revuelto, trabajando solo en un lugar que suele ser un laboratorio, con características semejantes a las de un laboratorio escolar (Chambers, 1983; Mead y Metraux, 1957). Y aunque la situación ha mejorado, ya que en los últimos años ha aumentado el porcentaje de mujeres dibujadas hasta el $28 \%$, son las niñas y niños más pequeños los que dibujan más mujeres, probablemente porque antes de empezar el colegio los estereotipos culturales que asocian a los hombres con la ciencia no han sido aprendidos y por eso no aparecen en sus dibujos (Miller y col., 2018; Ribera, 2018).

Por ello, como señala Vaillo (2016) "la solución al problema del sexismo y androcentrismo de los libros de texto no puede pasar por delegar todas las responsabilidades en manos de la voluntad y el buen hacer de las editoriales, o, peor aún, en base al criterio del profesorado". Es necesario que las administraciones, el Ministerio de Educación y las Comunidades Autónomas den 
los pasos necesarios para garantizar la presencia de mujeres en los libros de texto.

\section{Científicas e inventoras en los cuentos}

Para suplir las carencias que presentan los libros de texto y hacer visible la experiencia de las mujeres a lo largo de la Historia la Junta de Andalucía ha editado guías didácticas para el profesorado de Educación Primaria que con el nombre ¿Conoces a..? pretende dar a conocer a científicas como Hipatia, Ada Lovelace, y Marie Curie entre otras y que acompaña a las biografías de estas mujeres dirigidas al alumnado de primaria en formato cómic. Estas guías contienen una biografía más extensa de las protagonistas, propuestas de actividades y bibliografía para ampliar la información.

El libro Descubriendo a científicas editado por la Fundación Séneca nos muestra a 38 científicas e inventoras (Ada Lovelace, Hedy Lamarr, Marie Curie, Ángela Ruiz Robles...) a través de la mirada de niñas y niños que participaron en un taller de arte (Macho, 2015). También el Instituto de Ciencias Matemático elaboró dos catálogos $\mathrm{Mi}$ científica favorita y Mi científica favorita 2, con las biografías de 28 y 33 científicas respectivamente (Hipatia, Mary Anning, Marie Curie, Hedy Lamarr, Angeles Alvariño, Jane Goodall, Valentina Tereshkova, Wangari Maathai, Katherine Johnson, Maryam Mirzhakani, ... ) elegidas por estudiantes de $5^{\circ}$ y $6^{\circ}$ de primaria de veinticuatro centros españoles con el objetivo de "ofrecer al alumnado de primaria referencias de mujeres científicas, que quedan fuera de sus libros de texto, con las que poder identificarse. Además, ellas son 
modelos que no cumplen los estereotipos y, por tanto, los cuestionan "(Macho, 2018). Además, en la Guía de Recursos para \#Mujeres Tecnológicas, elaborada por la Fundación Mújeres Jóvenes, podemos encontrar una breve reseña biográfica de 9 mujeres (Ada Lovelace, Ángela Ruiz Robles, Hedy Lamarr,...) que han contribuido al desarrollo de la tecnología.

En esta misma línea, se elaboró una selección de científicas e inventoras que fue enviada a los centros de primaria que participaban en la segunda edición de la actividad Unha enxeñeira ou científica en cada cole. Esta selección estaba compuesta por mujeres que protagonizaban cuentos o relatos dirigidos al público juvenil y que han realizado importantes contribuciones a la ciencia y a la tecnología.

En los dos últimos años hemos visto aumentar considerablemente el número de libros o cuentos sobre mujeres científicas e inventoras. La publicación de libros como Las chicas son guerreras, Mujeres y Cuentos de buenas noches para niñas rebeldes, entre otros, nos permitieron realizar una selección de científicas e inventoras que fue enviada a los centros de primaria que participaban en la segunda edición de la actividad Unha enxeñeira ou científica en cada cole.

La selección de científicas e inventoras estaba compuesta por:

1. Hipatia (Alejandría, 370-415). Una de las primeras científicas de las que tenemos evidencias históricas. Matemática, filósofa y astrónoma, además de inventora. 
2. Mary Anning (Lyme Regis, 1799-1847). Paleontóloga que encontró los primeros fósiles de reptiles marinos de la época jurásica. Sus hallazgos fueron fundamentales para demostrar la extinción de los dinosaurios.

3. Ada Byron Lovelace (Londres, 1815-1852). Matemática y escritora, es la primera programadora informática de la historia. En su honor se celebra el día de Ada Lovelace, cuyo objetivo es reconocer el trabajo de las mujeres dedicadas a la ciencia, las matemáticas, la ingeniería y la tecnología.

4. Marie Curie (Varsovia, 1867- 1934). Pionera en el campo de la radiactividad. La primera persona en ganar dos Premios Nobel, en Física (1903) y Química (1911).

5. Ángela Ruiz Robles (Villamanín, 1895-1975). Maestra e inventora gallega precursora del libro electrónico y la tablet.

6. Hedy Lamarr (Viena, 1914-2000). Actriz e inventora de la tecnología que se utiliza en los GPS, en el Bluetooth y en las conexiones WiFi. En su honor se celebra el día del inventor cada 9 de noviembre.

7. Ánxeles Alvariño (Serantes, 1916-2005). Oceonógrafa y descubridora de 22 especies de organismos marinos. Es la primera científica gallega a la que dedicaron el Día de la Ciencia en Galicia.

8. Jane Goodall (Londres, 1934). Primatóloga, etóloga y antropóloga. Sus investigaciones sobre los chimpancés revolucionaron la primatología. Junto a ella trabaja la veterinaria gallega Rebeca Atencia.

9. Valentina Tereshkova (Malesnnikovo, 1937). Ingeniera y cosmonauta. Fue la primera mujer en volar al espacio., en la nave Vostok 6 el 16 de junio de 1963 dando cuarenta y ocho vueltas a la Tierra durante cerca de tres días. 
10. Wangari Maathai (Kenia, 1940-2011). Bióloga y primera mujer de África Oriental en recibir un doctorado. Galardonada con el Premio Nobel de la Paz. por su "contribución al desarrollo sostenible, a la democracia y a la paz".

Y en la tercera edición, tras la buena acogida de uno de los colegios participantes se volvió a enviar la selección añadiendo dos mujeres matemáticas:

11. Katherine Johnson (Virginia, 1918-). Matemática afroamericana que realizó los cálculos de las trayectorias de los primeros vuelos espaciales de Estados Unidos, entre ellos la del Apolo 11 a la Luna en 1969.

12. Maryam Mirzhakani (Teherán, 1977-2017). La primera mujer en ganar la Medalla Fields, considerada como el premio Nobel de las matemáticas.

Hay muchas mujeres que han contribuido al avance de la ciencia y tecnología, pero en esta selección sólo se muestran a las científicas y tecnólogas que protagonizan cuentos o relatos dirigidos al público juvenil. Además, se ha querido mostrar mujeres de distintas épocas, de distintas razas, de otros países y de la comunidad autónoma a quien iba dirigida esta selección (Galicia). En el anexo, para cada científica o inventora se muestran los libros seleccionados tanto para el público infantil, como para el profesorado, páginas web o blogs como Mujeres con ciencia, donde además de profundizar en su biografía podemos encontrar reseñas de libros y películas que nos permiten visibilizarlas (Calvo y Verdejo, 2017). Una lista que incluye algunos de los cuentos recopilados en el libro Mujeres Matemáticas: las grandes desconocidas (Verdejo, 2017) o en la página web de la iniciativa 11 de febrero (https://11defebrero.org/) y que se 
irá actualizando para incorporar las últimas publicaciones, como por ejemplo los recientes libros $O$ valor das ideas: $a$ precursora do libro electrónico, Nuevo Mundo. Isabel Zendal en la Expedición de la Vacuna o Mujeres 4.

Cuentos que nos hablan de niñas que soñaban en ir a África, que amaban las matemáticas, que recogían fósiles, que deseaban ser científicas no princesas y que promueven valores como la curiosidad o el amor y el respecto a la naturaleza. $Y$ que pueden servir para visibilizar a las científicas porque como señala López (2018) "ya sabemos mucho sobre lo que hicieron «ellos» y ahora toca hablar de "nosotras»". y cambiar la opinión del alumnado sobre la profesión científica. Recordemos que según estudios recientes (Pérez y de Pro, 2018) los chicos de primaria tienden a considerarla como una profesión de hombres. Una consideración que podríamos ayudar a cambiar con la lectura de estos cuentos ya que como señala Solís (2018)

"el trabajo con modelos científicos femeninos en los contenidos escolares no sólo es fundamental para que las niñas se sientan identificadas a la hora de construir su personalidad, ... sino también para los varones".

También pueden servir como complemento facilitando el aprendizaje de las ciencias o las matemáticas (Fernández, Harris y Aguirre, 2014), por ejemplo, complementando la enseñanza de las Ciencias naturales (los ecosistemas) con cuentos y preguntas mediadoras que fomentan y dirigen el debate (González y Martín, 2016).

\section{Conclusiones}

Los cuentos o relatos biográficos sobre mujeres científicas y tecnólogas pueden ayudarnos a combatir esa 
cultura androcéntrica que aún sigue presente en los manuales escolares y que invisibiliza las aportaciones femeninas al conocimiento y al desarrollo de nuestra sociedad. En esta comunicación, se presenta una selección de científicas y tecnólogas que el alumnado de primaria puede conocer con la lectura de cuentos o relatos, proporcionando de esta forma modelos femeninos en el ámbito científico-tecnológico.

Con estos cuentos y la realización de talleres impartidos por científicas y tecnólogas en los colegios se ha pretendido visibilizar a las mujeres que trabajan en el ámbito científicotecnológico, mostrando que hay una tradición, y que las mujeres pese a las limitaciones impuestas por nuestra sociedad han participado en este y otros ámbitos desde los inicios.

\section{Bibliografía}

Aguayo, E. y Calvo, E. (2017). Visibilizando a científicas e ingenieras en centros de Educación Primaria. En Cobos Sanchiz, D.; López-Meneses, E.; Martín Padilla, A. H.; Molina-García, L. y Jaén Martínez, A. (Eds.). INNOVAGOGÍA 2016. III Congreso Internacional sobre Innovación Pedagógica y Praxis Educativa. Libro de Actas. 28, 29 y 30 de noviembre de 2016. AFOE Formacion: Sevilla, (304-310). Recuperado de: http://hdl.handle.net/10347/15356.

Agudo, A. (2018). Lo que las niñas querrían ser de mayores si fueran niños. [en línea]. El Pais. Recuperado de: https://elpais.com/elpais/2018/06/10/planeta_futuro/1528 661907_969674.html.

Aladro, E., Padilla, G., Requeijo, P., Semova, D.J., García, J., García M.T. y Viñarás, M. (2014). La presencia y representación de la mujer científica en la prensa 
española. Revista Latina de Comunicación Social, 69, 176194.

Álvarez M. y Soneira G. (1991). Enseñanza y aprendizaje de las ciencias experimentales: la coeducación como meta, Memoria final de investigación, Madrid: CIDE.

Álvarez et al. (2017). O sexismo na linguaxe escrita e icónica en libros de texto: unha historia interminable. En A. González Penín, E. Aguayo Lorenzo, A. J. López Díaz (eds.) Transformando dende a Universidade XUGex, $V$ Xornada Universitaria Galega en Xénero (p. 237-246). Vigo: Universidade de Vigo.

Angulo, E. (2018). La prensa y las científicas. Recuperado de: https://mujeresconciencia.com/2018/02/14/la-prensa-lascientificas/

Bian, L., Leslie, S. J. y Cimpian, A. (2017). Gender stereotypes about intellectual ability emerge early and influence children's interests. Science, 355 (6323), 389391.

Blanco, N. (2000): El sexismo en los materiales educativos en la ESO, Instituto Andaluz de la Mujer, Sevilla.

Calvo et al. (2017). Unha enxeñeira ou científica en cada cole. En A. González Penín, E. Aguayo Lorenzo, A. J. López Díaz (eds.) Transformando dende a Universidade XUGex, V Xornada Universitaria Galega en Xénero (p. 95102). Vigo: Universidade de Vigo. Recuperado de: https://minerva.usc.es/xmlui/handle/10347/16838.

Calvo, E. y Verdejo, A. (2018). Literatura y cine para visibilizar a las científicas. En: Membiela, Pedro, Casado, Natalia, Cebreiros, Ma Isabel y Vidal, M. (Eds.). Nuevos desafíos en la enseñanza superior (pp. 101-1105). Ourense: Educación Editora.

Chambers, D. W. (1983). Stereotypic images of the scientist:

The Draw-a-Scientist Test. Science education, 67(2), 255265.

Couso, D. (2015). Vocaciones científicas: muchas soluciones y 
ninguna simple; ¿qué podríamos hacer y no hacemos?. Jornada sobre experiencias en promoción de vocaciones científicas y tecnológicas. Obra social La Caixa-Fecyt. Cosmocaixa (Barcelona).

Fernández, A. B. e Ibáñez, M. (2018). Más mujeres en los estudios de Informática: una propuesta desde el departamento de formación y orientación laboral. RASE: Revista de la Asociación de Sociología de la Educación, 11(1), 116-134.

Fernández, R., Harris, C., y Aguirre, C. (2014). Propuestas para el tratamiento de la Competencia Matemática y de Ciencias a través de la literatura infantil en Educación Infantil y Primaria. Números. Revista de Didáctica de las Matemáticas, 85, 25-39.

García Nieto, M. T. (2013) ¿Son invisibles las mujeres científicas?. Estudios sobre el Mensaje Periodístico, 19, 783-792.

González, D., Mateu, A., Pons, E. y M. Domínguez (2017). Women scientists as decor: The image of scientists in spanish press pictures. Science Communication, 39, 4, 535-547.

Gutiérrez Pereda, I. (2017). Mujeres inventoras, mujeres ingenieras. Recuperado de: https://repositorio.unican.es/xmlui/handle/10902/13130.

Hamodi, C. (2014). ¿Transmiten los libros de texto el valor de la igualdad desde la perspectiva de género? estudio del lenguaje icónico de dos editoriales. REIRE, 7, 1, 30-55.

Hill, C., Corbett, C. y A. Rose (2010). Why So Few? Women in Science, Technology, Engeneering and Mathematics. Washington, DC: AAUW. Recuperado de http://files.eric.ed.gov/fulltext/ED509653.pdf.

Lomas, C. (2002). El sexismo en los libros de texto. En Ana González y Carlos Lomas (coords.). Mujer y educación: educar para la igualdad, educar desde la diferencia. Barcelona: Graó. 
López-Navajas, A. (2014). Análisis de la ausencia de las mujeres en los manuales de la ESO: una genealogía de conocimiento ocultada", Revista de Educación, 363, 282308.

Macho, M. (2015). Descubriendo científicas. Recuperado de: https://mujeresconciencia.com/2015/10/16/descubriendocientificas/

Macho, M. (2016). Marie-Anne Pierrette Paulze, la 'madre de la química moderna'. Recuperado de: https://mujeresconciencia.com/2016/01/20/marie-annepierrette-paulze-la-madre-de-la-quimica-moderna/

Macho, M. (2018). Mi científica favorita. Recuperado de: https://mujeresconciencia.com/2018/01/05/mi-cientificafavorita/

Manassero, M. A., y Vázquez, Á. (2003). Las mujeres científicas: un grupo invisible en los libros de texto. Revista Investigación en la Escuela, (50), 31-45.

Mead, M., y Metraux, R. (1957). Image of the scientist among high-school students. Science, 126(3270), 384390.

Miller, D. I., Nolla, K. M., Eagly, A. H. and Uttal, D. H. (2018), The Development of Children's Gender-Science Stereotypes: A Meta-analysis of 5 Decades of U.S. Draw-A-Scientist Studies. Child Dev. https://doi.org/10.1111/cdev.13039

Navarro Marco, S. (2016). Análisis del papel de la mujer en la ciencia y su transmisión durante la Educación Primaria. Recuperado de: http://uvadoc.uva.es/handle/10324/18694.

Pérez, A. y de Pro, A. (2018). Algunos datos sobre la visión de los niños y de las niñas sobre las ciencias y del trabajo científico. iQual. Revista de Género e Igualdad, (1), 18-31

Pinedo, R., Arroyo, M. J. y Berzosa, I. (2018). Género y educación: detección de situaciones de desigualdad de género en contextos educativos. Contextos Educativos. 
Revista de Educación, (21), 35-51.

Ribera, A. (2018). Dibuja un científico. Recuperado de: https://mujeresconciencia.com/2018/04/11/dibuja-uncientifico/

Rodríguez-Pina, G. (2018). El "conocimiento amputado" de los libros de texto sin mujeres. [en línea]. El Pais. Recuperado de: https://elpais.com/politica/2018/07/07/actualidad/153095 2055_654127.html.

Saéz, M. J. y Clavero, N. (2016). Hipatia de Alejandría: La Dama de las Ciencias. Una propuesta interdisciplinar en primaria a través del uso de textos. Revista Eureka sobre Enseñanza y Divulgación de las Ciencias, 13(3), 628-642.

Solis-Espallargas, C. (2018) Inclusión del enfoque de género en la enseñanza de las ciencias mediante el estudio de biografías de mujeres científicas. Revista Eureka sobre Enseñanza y Divulgación de las Ciencias 15(3), 3602.

Subirats, M. (1993): El sexismo en los libros de texto: análisis y propuesta de un sistema de indicadores, Ministerio de Asuntos Sociales, Madrid.

Vaillo, M. (2016). La investigación sobre libros de texto desde la perspectiva de género: ¿hacia la renovación de los materiales didácticos? Tendencias pedagógicas, 27, pp. 97-124.

Verdejo, A. (2017). Mujeres matemáticas: las grandes desconocidas. Vigo: Universidade de Vigo, Servizo de Publicacións.

\section{Cómo referenciar este artículo/How to reference this article:}

Calvo Iglesias, M.E. (2019). Científicas e inventoras a través de los cuentos. iQUAL. Revista de Género e lgualdad, 2, 147-170, doi: 10.6018/iQual.340701

Calvo Iglesias, M.E. (2019). Científicas e inventoras a través de los cuentos. [Female scientists and inventors through tales]. iQUAL. Revista de Género e Igualdad, 2, 147-170, doi: 10.6018/iQual.340701 


\section{Anexo}

\section{HIPATIA (Alejandría, 370-415) Una de las primeiras científicas de las} que tenemos evidencias históricas.

\section{Unidad didáctica para 5 de Primaria:}

Sáez Bondia, Mª J., Clavero Pagés, N. (2016). Hipatia de Alejandría: La Dama de las Ciencias. Una propuesta interdisciplinar en primaria a través del uso de textos. Revista Eureka sobre Enseñanza y Divulgación de las Ciencias, 13 (3), 628-642. Recuperado de: http://hdl.handle.net/10498/18502
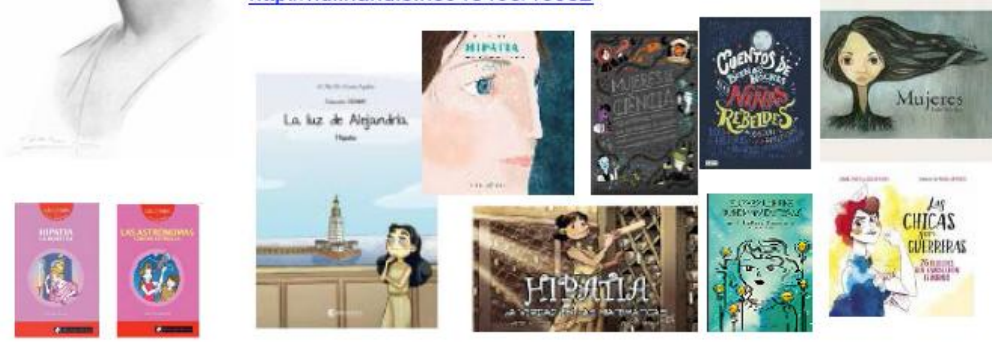

\section{LIBROS}

- Salesas i Pla, Florenci (2009) HIPATIA la maestra. Madrid: El Rompecabezas (Colección Sabelotod@s). A partires de 9 anos.

- Gil, Ma Pilar; Aguilera, Cristina (ilustradora) (2015): La luz de Alejandría. Hipatia. EditorialSalvatella (Colección Genius).

- Bayarri, Jordi y Seijas, Dani (2018). Hipatia. La verdad en las matemáticas. Editorial: Jordi Bayarri

- Garcia Tur, Victor (2018). Hipatia, la gran maestra de Alejandría. Barcelona: Vegueta Ediciones.

También aparece junto a otras científicas en:

- Gil Casanova, Sara (2014). LAS ASTRÓNOMAS, chicas estrella. Madrid: EI Rompecabezas (Colección Sabelotod@s). A partires de 9 anos.

- Ruiz Ruiz, Isabel (2015). Mujeres. Madrid:llustropos.

- Cívico, Irene; Parra, Sergio; Aparicio, Nuria (ilustradora) (2016). Las chicas son guerreras. $26 \mathrm{R}$ ebeldes que cambiaron el mundo. Montena. Páxina 12-15.

- Favilli, Elena y Cavallo, Francesca (2017). Cuentos de buenas noches para niñas rebeldes. Destino. Páxina: 82-83.

- Ignotofsky, Rachel (2017). Mujeres de ciencia. Nórdica libros. Páginas 9-10.

- Uve, Sandra (2018). Supermujeres, superinventoras. Ideas brillantes que transformaron nuestra vida. Planeta. Páxina 102.

\section{Página web: http://mujeresconciencia.com/2015/06/15/hipatia/}

EXPOSICIONES: Con A de Astrónomas http://www.sea-astronomia.es/drupal/?q=node/1163

Mujeres Tecnólogas https://blogs.deusto.es/mujertekspace/expo/ 


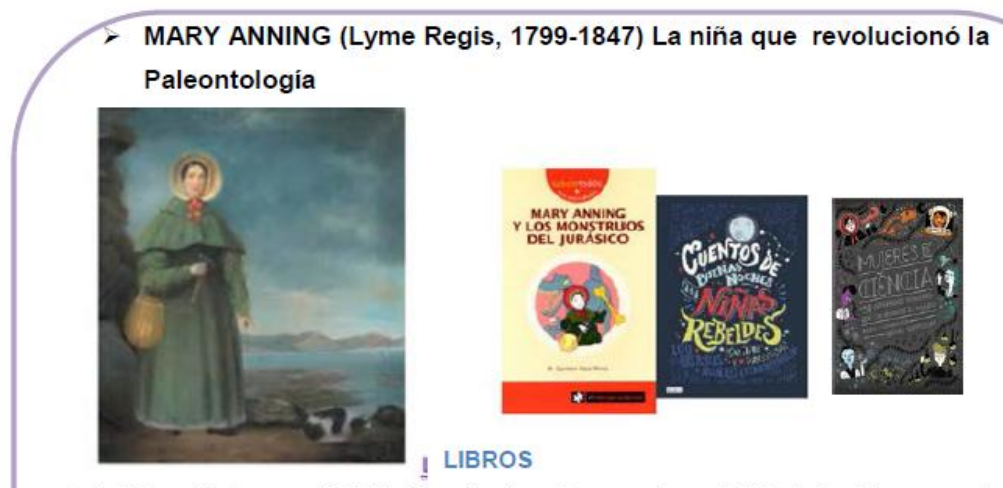

-Ruiz Pérez, M. Carmen (2012). Mary Anning y los monstruos del Jurásico. El rompecabezas (a partires de 9 anos).

También aparece xunto con outras científicas en:

- Favilli, Elena y Cavallo, Francesca (2017). Cuentos de buenas noches para niñas rebeldes. Destino. Páginas: 136-137.

-Ignotofsky, Rachel (2017). Mujeres de ciencia. Nórdica libros. Páginas 15-16.

Páginas web:

http://www.bbc.co.uk/schools/primaryhistory/famouspeople/mary anning/

http://mujeresconciencia.com/2014/05/21/mary-anning-buscadora-de-fosiles/

Para el profesorado Chevalier, Tracy (2010). Las huellas de la vida, Lumen.

ADA BYRON LOVELACE (Londres, 1815-1852 ). La primera programadora de la historia.
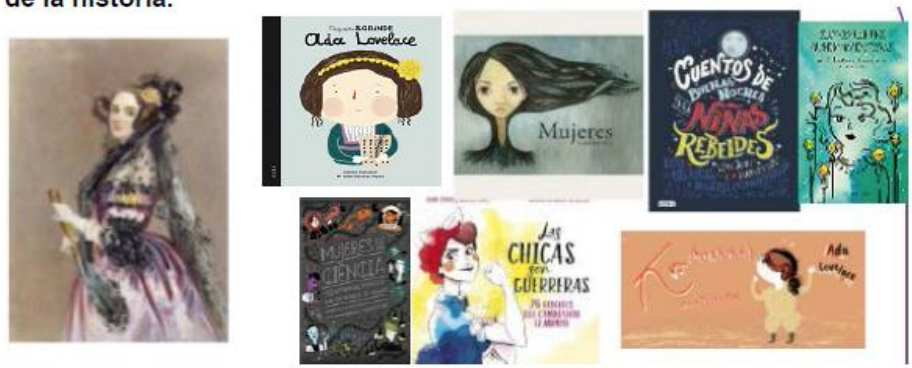

LIBROS

- Ruiz Ruiz, Isabel (2015) : Mujeres 2. Madrid: Ilustropos Sánchez Vergara, Isabel (2018). Pequeña\&Grande. Ada Lovelace. Alba Editorial.

- Civico, Irene; Parra, Sergio; Aparicio, Nuria (ilustradora) (2016) Las chicas son guerreras. 26 rebeldes que cambiaron el mundo. Montena. Páxina 20-23.

- Favilli, Elena y Cavallo, Francesca (2017). Cuentos de buenas noches para niñas rebeldes. Destino. Páxina: 14-15. 
Material recopilado por Encina Calvo Iglesias

- Ignotofsky, Rachel (2017). Mujeres de ciencia. Nórdica libros. Páginas 17-18.

- Uve, Sandra (2018). Supermujeres, superinventoras. Planeta. Páxina 1

- Un cuento propio. Pandora Mirabilia http://biodiversiacoop.net/wpcontent/uploads/2017/04/taller-ada-lovelace.pdf

Páginas web: Podemos encontrar una minibiografia en las seguintes páginas:

http://rapacinas.gal/ada-byron

http://fseneca.es/descubriendo-cientificas/ada-lovelace/

http://mujeresconciencia.com/2014/12/10/ada-lovelace-pionera-en-programacion

Para el profesorado

Essinger, James (2015). El algoritmo de Ada. La vida de Ada Lovelace, hija de lord Byron y pionera de la era informática. Alba Editorial.

MARIE CURIE (Varsovia, 1867- 1934 ). La primera persona en ganar dos Premios NOBEL

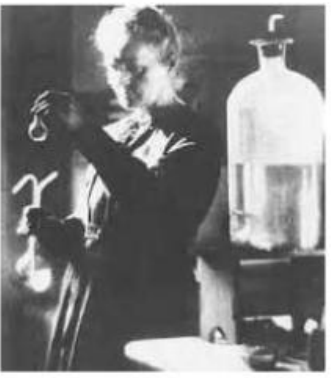

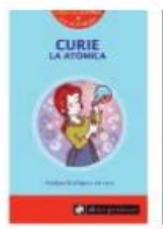
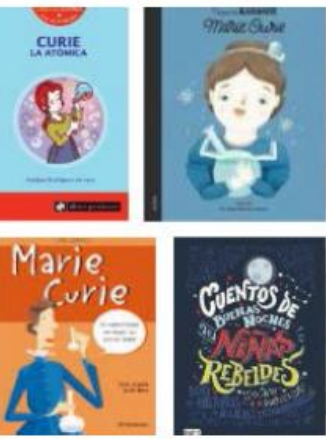
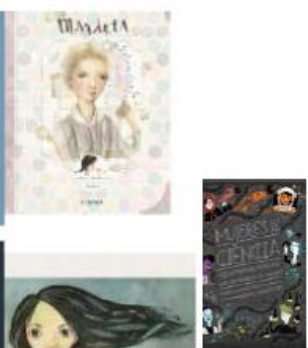

LIBROS

- Rodríguez Serrano, Esteban (2010). Curie la atómica. Editorial Susaeta

- Cugota, Lluis; Vera, Luisa (2014). Marie Curie. Editorial Parramón (Colección Me llamo : ... ).

- Miranda Vicente, Itziar; Miranda Vicente, Jorge (2015). Marieta. Editorial Edelvives.

-Sánchez Vergara, Isabel (2016). Pequeña\&Grande. Marie Curie. Alba Editorial.

Tamén aparece en:

-Ruiz Ruiz, Isabel (2015): Mujeres. Madrid: Ilustropos.

- Cívico, Irene; Parra, Sergio; Aparicio, Nuria (ilustradora) (2016): Las chicas son guerreras. 26 rebeldes que cambiaron el mundo. Montena. Páxina 28.

- Favilli, Elena y Cavallo, Francesca (2017). Cuentos de buenas noches para niñas rebeldes.

Destino. Páxina: 134-35.

-Ignotofsky, Rachel (2017). Mujeres de ciencia. Nórdica libros. Páginas 17-18.

Páginas web:

http://mujeresconciencia.com/2014/11/07/maria-curie-pionera-en-radiactividad/

http://fseneca.es/descubriendo-cientificas/marie-curie/c

Para el profesorado: Montero, Rosa (2013). La ridicula idea de no volver a verte. Seix Barral 


\section{- ÁNGELA RUIZ ROBLES (Villamanín, 1895 - 1975). La maestra e inventora} gallega precursora del e-book.
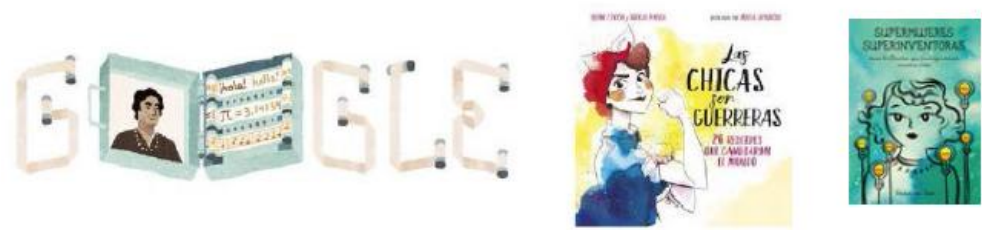

\section{LIBROS}

Su biografía aparece en

-Cívico, Irene; Parra, Sergio; Aparicio, Nuria (ilustradora) (2016): Las chicas son guerreras. 26 rebeldes que cambiaron el mundo. Montena. Páxina 60-63.

-Uve, Sandra (2018). Supermujeres, superinventoras. Planeta. Páxina 26.

Páginas web:

Podemos encontrar una minibiografía en

http://rapacinas.gal/angela-ruiz

http://fseneca.es/descubriendo-cientificas/angela-ruiz-robles/

http://mujeresconciencia.com/2016/03/28/angela-ruiz-robles-maestra-e-inventora/

http://www.nomecuentescuentos.com/angela-ruiz-roblez-la-precusora-del-libro/

\section{HEDY LAMARR (Viena, 1914-2000). Actriz e inventora que imaginó la} wifi.
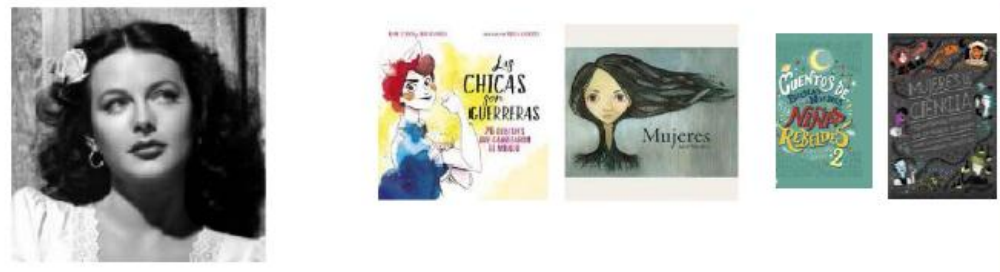

\section{LIBROS}

-Ruiz Ruiz, Isabel (2015): Mujeres 2. Madrid: llustropos.

-Civico, Irene; Parra, Sergio; Aparicio, Nuria (ilustradora) (2016): Las chicas son guerreras. 26 rebeldes que cambiaron el mundo. Montena. Páxina 84-63.

-Ignotofsky, Rachel (2017). Mujeres de ciencia. Nórdica libros. Páginas 69-70.

-Favilli, Elena y Cavallo, Francesca (2018). Cuentos de buenas noches para niñas rebeldes 2. Destino.

Páginas web: Podemos encontrar una minibiografía en http://fseneca.es/descubriendo-cientificas/hedy-lamarr/ http://mujeresconciencia.com/2014/11/09/hedy-lamarr-inventora/ 
- ÁNXELES ALVARIÑO (Serantes, 1916 - 2005). La primeira científica gallega A la que dedicaron $O$ día da Ciencia en Galicia en 2015.
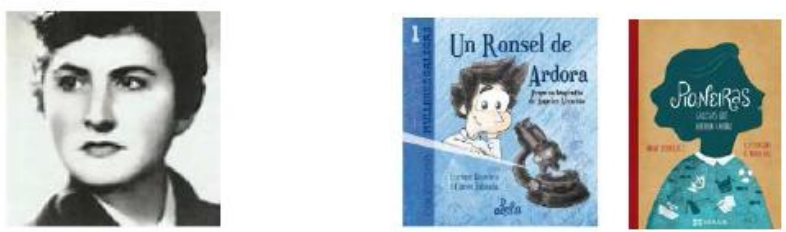

LIBROS

Mauricio, Enrique; Taboada, Carlos (2016). Un ronsel de ardora. Pequena biografía de Ánxeles Alvariño. Lela edicións.

Rodriguez, Anair (2018). Pioneiras. Editorial Xerais.

Páginas web:

http://mujeresconciencia.com/2016/09/19/angeles-alvarino-la-gran-oceanografa/

\section{KATHERINE JOHNSON (1918-) La niña que amaba contar.}
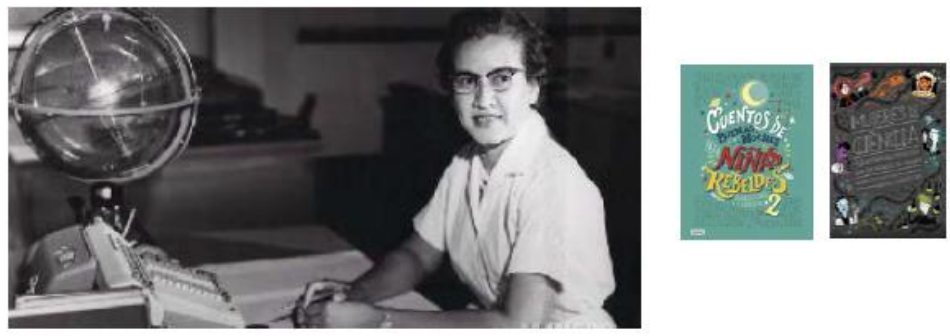

\section{Libros}

- Ignotofsky, Rachel (2017). Mujeres de ciencia. Nórdica libros. Páginas 74-75.

- Favilli, Elena y Cavallo, Francesca (2018). Cuentos de buenas noches para niñas rebeldes 2. Destino.

Páginas web:

-https://www.beyondcurie.com/katherine-johnson/

-https://mujeresconciencia.com/2018/03/02/she-changed-stem/

Para el profesorado: La película Fíguras Ocultas

Melfi, T. (2016). Figuras Ocultas. USA: Levantine Films / Chernin Entertainment / Fox 2000 Pictures. 


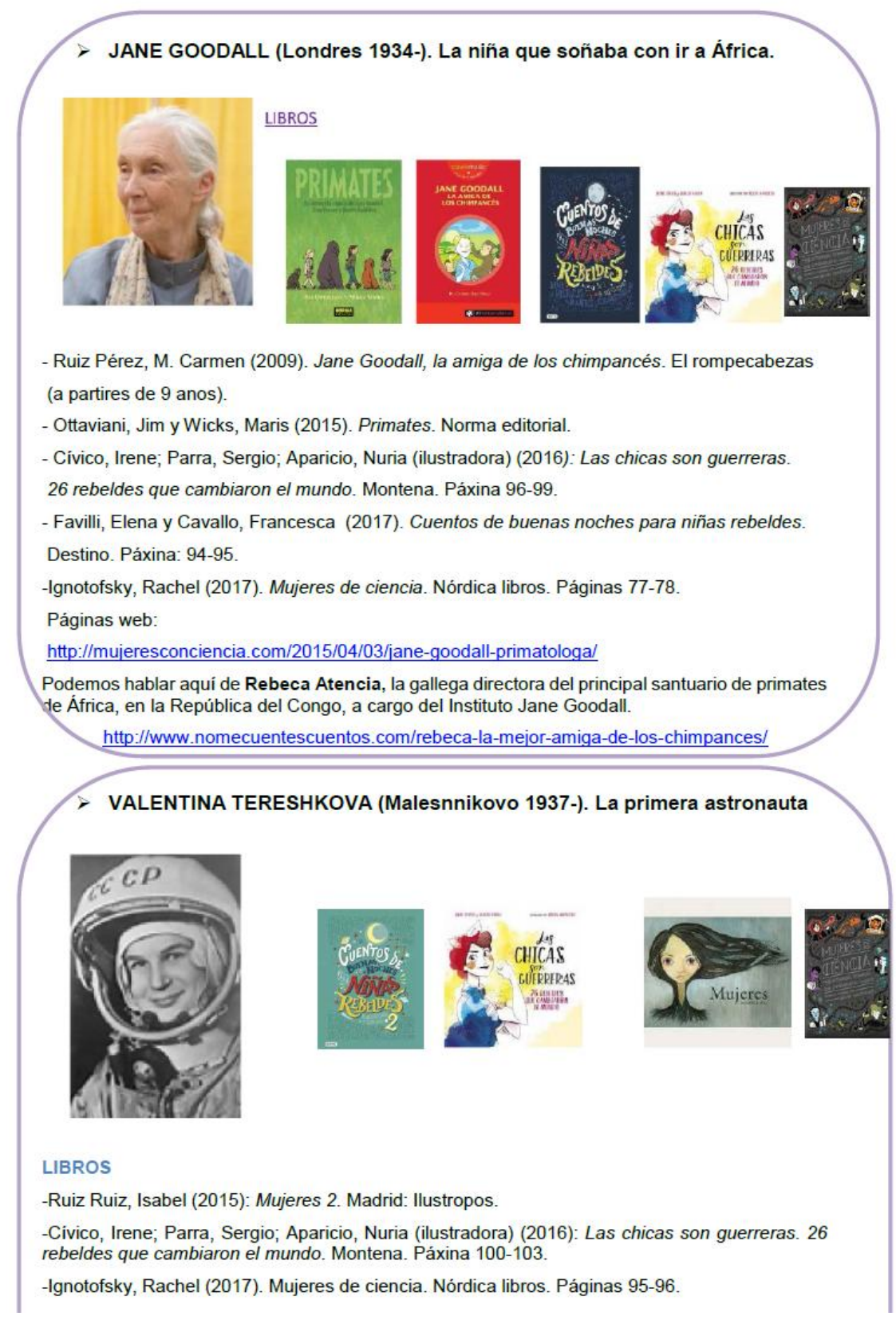


-Favilli, Elena y Cavallo, Francesca (2018). Cuentos de buenas noches para niñas rebeldes 2 Destino.

Páginas web: http://mujeresconciencia.com/2016/01/04/valentina-vladimirovna-tereshkova-laprimera-viajera-espacial/

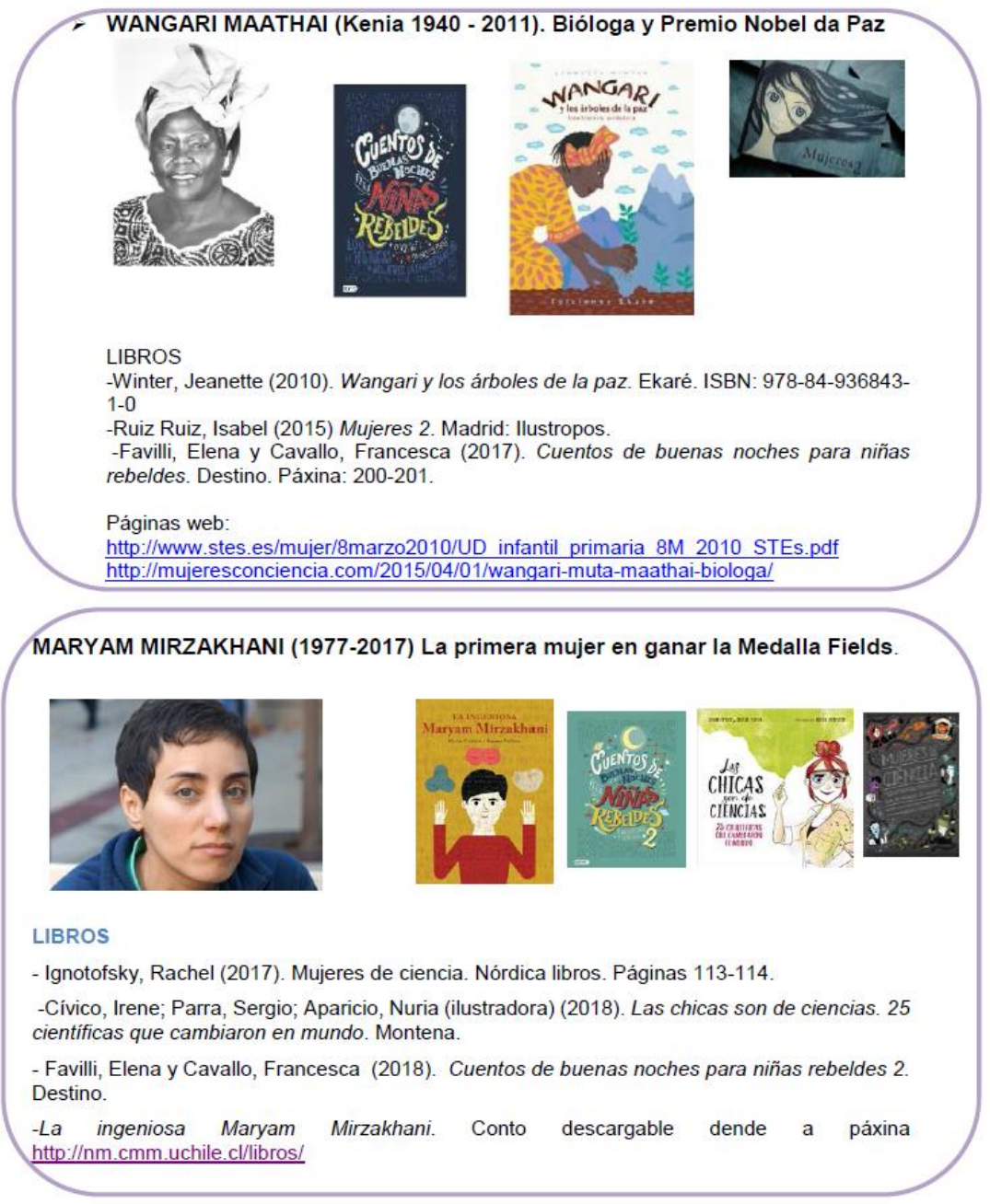

